\title{
HOLOMORPHIC MORSE INEQUALITIES
}

Jean-Pierre DEMAILLY, Université de Grenoble I

Series of Lectures given at the AMS Summer Institute held in Santa Cruz, California, July 1989.

\section{Introduction}

Let $M$ be a compact $C^{\infty}$ manifold, $\operatorname{dim}_{\mathbb{R}} M=m$, and $h$ a Morse function, i.e. a function such that all critical points are non degenerate. The standard Morse inequalities relate the Betti numbers $b_{q}=\operatorname{dim} H_{D R}^{q}(M, \mathbb{R})$ and the numbers

$$
s_{q}=\# \text { critical points of index } q,
$$

where the index of a critical point is the number of negative eigenvalues of the Hessian form $\left(\partial^{2} h / \partial x_{i} \partial x_{j}\right)$. Specifically, the following "strong Morse inequalities" hold :

$$
b_{q}-b_{q-1}+\cdots+(-1)^{q} b_{0} \leqslant s_{q}-s_{q-1}+\cdots+(-1)^{q} s_{0}
$$

for each integer $q \geqslant 0$. As a consequence, one recovers the "weak Morse inequalities" $b_{q} \leqslant s_{q}$ and the expression of the Euler-Poincaré characteristic

$$
\chi(M)=b_{0}-b_{1}+\cdots+(-1)^{m} b_{m}=s_{0}-s_{1}+\cdots+(-1)^{m} s_{m} .
$$

The purpose of these lectures is to explain what are the complex analogues of these inequalities for $\bar{\partial}$-cohomology groups with values in holomorphic line (or vector) bundles, and to present a few applications.

Let $X$ be a compact complex manifold, $n=\operatorname{dim}_{\mathbb{C}} X$ and $E, F$ holomorphic vector bundles over $X$ with

$\operatorname{rank} E=1, \operatorname{rank} F=r$.

We denote here $h^{q}(F)=\operatorname{dim} H^{q}(X, \mathcal{O}(F))$. 
Assume that $E$ is endowed with a $C^{\infty}$ hermitian metric and denote by $c(E)$ its curvature form. Then $i c(E)$ is a real $(1,1)$-form on $X(c f . \S 2)$. Finally, consider the $q$-index sets

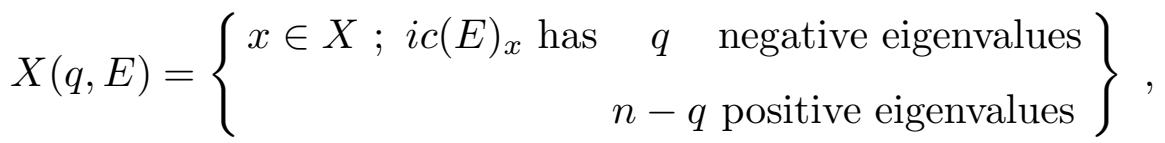

$$
\begin{aligned}
& X(\leqslant q, E)=\bigcup_{1 \leqslant j \leqslant q} X(j, E) .
\end{aligned}
$$

Observe that $X(q, E)$ and $X(\leqslant q, E)$ are open subsets of $X$.

Main Theorem. - The sequence of tensor powers $E^{k} \otimes F$ satisfy the following asymptotic estimates as $k \rightarrow+\infty$ :

(1.3) Weak Morse inequalities :

$$
h^{q}\left(E^{k} \otimes F\right) \leqslant r \frac{k^{n}}{n !} \int_{X(q, E)}(-1)^{q}\left(\frac{i}{2 \pi} c(E)\right)^{n}+o\left(k^{n}\right) .
$$

(1.4) Strong Morse inequalities :

$$
\sum_{0 \leqslant j \leqslant q}(-1)^{q-j} h^{j}\left(E^{k} \otimes F\right) \leqslant r \frac{k^{n}}{n !} \int_{X(\leqslant q, E)}(-1)^{q}\left(\frac{i}{2 \pi} c(E)\right)^{n}+o\left(k^{n}\right) .
$$

(1.5) Asymptotic Riemann-Roch formula :

$$
\chi\left(E^{k} \otimes F\right)=r \frac{k^{n}}{n !} \int_{X}\left(\frac{i}{2 \pi} c(E)\right)^{n}+o\left(k^{n}\right) .
$$

Observe that (1.5) is in fact a weak consequence of the Hirzebruch-RiemannRoch formula.

The above theorem was first proved in [De 2] in 1985. It was largely motivated by Siu's solution of the Grauert-Riemenschneider conjecture ([Siu 2], 1984), which we will reprove below as a special case of a stronger statement. The basic tool is a spectral theorem which describes the eigenvalue distribution of the complex Laplace-Beltrami operators. The original proof of [De 2] was based partly on Siu's techniques and partly on an extension of Witten's analytic proof [Wi, 1982] for the standard Morse inequalities. Somewhat later Bismut [Bi] and quite recently Getzler [Ge 3] gave new proofs, both relying on an analysis of the heat kernel in the spirit of Atiyah-Bott-Patodi's proof of the Atiyah-Singer index theorem [A-B-P]. Although the basic idea is simple, Bismut used deep results arising from probability theory (the Malliavin calculus), while Getzler relied on his supersymmetric symbolic calculus for spin pseudodifferential operators [Ge 1].

We will try to present here a simple heat equation proof, based essentially on Mehler's formula and elementary asymptotic estimates (cf. $\S 3$ and $\S 4$ ). The next sections deal with various generalizations and applications : 
- in $\S 5$, we obtain as a consequence a strong characterization of Moishezon varieties in terms of the existence of line bundles satisfying some integral positivity hypothesis of the curvature. In particular, this gives a solution of the GrauertRiemenschneider conjecture. We also give a generalization to all projective algebraic manifolds of G. Kempf's distortion inequalities for ample line bundles over abelian varieties [Kem].

- in $\S 6$, the case of $q$-convex manifolds is considered. As shown by Thierry Bouche [Bou 1], Morse inequalities then hold in degrees $\geqslant q$. By an argument of Siu [Siu 3,4], these inequalities imply a general a priori estimate for the MongeAmpère operator.

- in $\S 7$, we present (with a different and more elementary proof) Getzler's extension [Ge 1] of holomorphic Morse inequalities to the vector bundle case.

- $\S 8$ briefly discusses the degenerate case when $i c(E)$ has $\operatorname{rank}<\operatorname{dim} X$ everywhere, as well as related open problems.

\section{Hermitian connections, curvature and Laplace-Beltrami operators}

Let $F$ be a complex vector bundle of rank $r$ over a smooth differentiable manifold $M$. We denote by $C_{q}^{\infty}(M, F)$ the space of smooth $q$-forms with values in $F$, i.e. smooth sections of $\Lambda^{q} T^{*} M \otimes F$. To fix notations, we recall here the basic terminology used.

A connection $D$ on $F$ is a linear differential operator

$$
D: C_{q}^{\infty}(M, F) \rightarrow C_{q+1}^{\infty}(M, F)
$$

such that

$$
D(f \wedge u)=d f \wedge u+(-1)^{\operatorname{deg} f} f \wedge D u
$$

for all forms $f \in C_{p}^{\infty}(X, \mathbb{C}), u \in C_{q}^{\infty}(X, F)$. On an open set $\Omega \subset M$ where $F$ is trivial, $F_{\mid \Omega} \simeq \Omega \times \mathbb{C}^{r}$, a connection $D$ can be written

$$
D u=d u+\Gamma \wedge u
$$

where $\Gamma \in C_{1}^{\infty}\left(\Omega, \operatorname{Hom}\left(\mathbb{C}^{r}, \mathbb{C}^{r}\right)\right)$ is an arbitrary matrix of 1 -forms and $d$ acts componentwise. It is then easy to check that

$$
D^{2} u=(d \Gamma+\Gamma \wedge \Gamma) \wedge u \text { on } \Omega,
$$

so that $D^{2} u=c(D) \wedge u$ for some global 2-form

$$
c(D) \in C_{2}^{\infty}(M, \operatorname{Hom}(F, F))
$$

called the curvature of $D$.

Assume now that $F$ is endowed with a $C^{\infty}$ hermitian metric along the fibers and that the isomorphism $F_{\mid \Omega} \simeq \Omega \times \mathbb{C}^{r}$ is given by a $C^{\infty}$ frame $\left(e_{\lambda}\right)$. We then have a canonical sesquilinear pairing

$$
\begin{array}{ccc}
C_{p}^{\infty}(M, F) & \longrightarrow & C_{p+q}^{\infty}(M, \mathbb{C}) \\
(u, v) & \longmapsto & \{u, v\}
\end{array}
$$


given by

$$
\{u, v\}=\sum_{\lambda, \mu} u_{\lambda} \wedge \bar{v}_{\mu}\left\langle e_{\lambda}, e_{\mu}\right\rangle \quad \text { for } u=\sum u_{\lambda} \otimes e_{\lambda}, v=\sum v_{\mu} \otimes e_{\mu} .
$$

The connection $D$ is said to be hermitian if it satisfies the additional property

$$
d\{u, v\}=\{D u, v\}+(-1)^{\operatorname{deg} u}\{u, D v\} .
$$

Assuming that $\left(e_{\lambda}\right)$ is orthonormal, one easily checks that $D$ is hermitian if and only if $\Gamma^{*}=-\Gamma$. In this case $c(D)^{*}=-c(D)$, thus

$$
i c(D) \in C_{2}^{\infty}(M, \operatorname{Herm}(F, F)) \text {. }
$$

(2.3) Special case. For a bundle $E$ of rank 1 , the connection form $\Gamma$ of a hermitian connection $D$ can be written $\Gamma=-i A$ where $A$ is a real $1-$ form. Then we have $c(D)=d \Gamma=-i d A$ and we will denote

$$
B=i c(D)=d A
$$

A phase change $u=v e^{i \theta}$ in the isomorphism $E_{\mid \Omega} \simeq \Omega \times \mathbb{C}$ replaces $A$ by the new connection form $A+d \theta$.

(2.4) Complex analytic case. If $M$ is a complex manifold $X$, every connection $D$ can be splitted in a unique way as a sum of a $(1,0)$ and of a $(0,1)$-connection :

$$
D=D^{\prime}+D^{\prime \prime} \text {. }
$$

In a local trivialization given by a $C^{\infty}$ frame, one can write

$$
\begin{aligned}
D^{\prime} u & =d^{\prime} u+\Gamma^{\prime} \wedge u, \\
D^{\prime \prime} u & =d^{\prime \prime} u+\Gamma^{\prime \prime} \wedge u,
\end{aligned}
$$

with $\Gamma=\Gamma^{\prime}+\Gamma^{\prime \prime}$. The connection is hermitian if and only if $\Gamma^{\prime}=-\left(\Gamma^{\prime \prime}\right)^{*}$ in any orthonormal frame. Thus there exists a unique hermitian connection corresponding to a prescribed $(0,1)$ part $D^{\prime \prime}$.

Assume now that the bundle $E$ itself has a holomorphic structure. The unique hermitian connection for which $D^{\prime \prime}=\bar{\partial}$ is called the Chern connection of $F$. In a local holomorphic frame $\left(e_{\lambda}\right)$ of $E_{\mid \Omega}$, the metric is given by some hermitian matrix $H=\left(h_{\lambda \mu}\right)$ where $h_{\lambda \mu}=\left\langle e_{\lambda}, e_{\mu}\right\rangle$. Easy computations yield the expression of the Chern connection :

$$
\left\{\begin{array}{l}
D^{\prime} u=\partial u+\bar{H}^{-1} \partial \bar{H} \wedge u \\
d^{\prime \prime} u=\bar{\partial} u \\
c(F) \underset{\text { def }}{=} c(D)=-\bar{\partial}\left(\bar{H}^{-1} \partial \bar{H}\right)
\end{array}\right.
$$

For a rank 1 bundle $E$, the matrix $H$ is simply a positive weight function $e^{-\varphi}$, $\varphi \in C^{\infty}(\Omega, \mathbb{R})$, and we find

$$
c(E)=\partial \bar{\partial} \varphi
$$


(2.5) Hodge theory. Assume now that $M$ is a riemannian manifold with metric $g=\sum g_{i j} d x_{i} \otimes d x_{j}$. Given a $q$-form $u$ on $M$ with values in $F$, we consider the global $L^{2}$ norm

$$
\|u\|^{2}=\int_{M}|u(x)|^{2} d \sigma(x)
$$

where $|u|$ is the pointwise hermitian norm and $d \sigma$ the riemannian volume form. The Laplace Beltrami operator associated to the connection $D$ is

$$
\Delta=D D^{*}+D^{*} D
$$

where $D$ is the (formal) adjoint of $D$; the complex Laplace operators $\Delta^{\prime}$ and $\Delta^{\prime \prime}$ are defined similarly. In degree 0 we simply have $\Delta=D^{*} D$.

When $M$ is compact, the elliptic operator $\Delta$ has a discrete spectrum

$$
\lambda_{1} \leqslant \lambda_{2} \leqslant \cdots \leqslant \lambda_{j} \leqslant \cdots
$$

and corresponding eigenfunctions $\psi_{j} \in C_{q}^{\infty}(M, F) \cap L^{2}$. Our main goal is to obtain asymptotic formulae for the eigenvalues. For that, we make use of the heat operator $e^{-t \Delta}$. In the above setting, the heat operator is the bounded hermitian operator associated to the heat kernel

$$
K_{t}(x, y)=\sum_{j=1}^{+\infty} e^{-\lambda_{j} t} \psi_{j}(x) \otimes \psi_{j}^{*}(y),
$$

and $K_{t} \in C^{\infty}(] 0,+\infty[\times M \times M, \operatorname{Hom}(F, F))$. The asymptotic distribution of eigenvalues can be recovered from the well known (obvious) formula

$$
\sum_{j=1}^{+\infty} e^{-\lambda_{j} t}=\int_{M} \operatorname{tr}_{F} K_{t}(x, x) d \sigma(x) .
$$

\section{Asymptotic formulas for the heat kernel and the eigenvalue distribution}

Let $E, F \rightarrow M$ be complex vector bundles equipped with hermitian connections, $D_{k}$ the associated connection on $E^{k} \otimes F$ and $\Delta_{k}=D_{k}^{*} D_{k}$ the Laplace-Beltrami operator acting on sections of $E^{k} \otimes F$ (degree 0). Finally, let $V \in C^{\infty}(M, \operatorname{Herm}(F, F))$; we still denote $V$ the operator $\operatorname{Id}_{E^{k}} \otimes V$ acting on $E^{k} \otimes F$.

If $\Omega \subset \subset M$ is a smoothly bounded relatively compact open subset of $M$, we consider the quadratic form

$$
Q_{k, \Omega}(u)=\int_{\Omega} \frac{1}{k}\left|D_{k} u\right|^{2}-\langle V u, u\rangle
$$

with domain equal to the Sobolev space $W_{0}^{1}\left(\Omega, E^{k} \otimes F\right)=$ closure of the space of smooth sections with compact support in $\Omega$, in the space $W_{\text {loc }}^{1}\left(M, E^{k} \otimes F\right)$ of 
sections which have $L_{\text {loc }}^{2}$ coefficients as well as their first derivatives. That is, we consider the self adjoint operator

$$
\square_{k}=\frac{1}{k} D_{k}^{*} D_{k}-V
$$

densely defined in the Hilbert space $W_{0}^{1}\left(\Omega, E^{k} \otimes F\right)$ (Dirichlet boundary conditions).

We want to study the asymptotic eigenvalue distribution of $\square_{k}$ as $k \rightarrow+\infty$, as well as an asymptotic formula for the heat kernel $e^{-t \square_{k}}$. The basic idea is extremely simple and reduces the proof into two steps :

- show that the asymptotic estimates are purely local (up to error terms of lower order) and can be obtained by freezing the coeffiicents of the operators involved at any given point.

- compute explicitly the heat kernel in the case of connections with constant curvature, assuming that $M$ has a flat metric.

In order to see that the situation is local, let $\left(\psi_{j}\right)$ be a partition of unity relative to an arbitrarily fine covering of $\bar{\Omega}$, such that $\sum \psi_{j}^{2}=1$ near $\bar{\Omega}$. As $\sum \psi_{j} d \psi_{j}=0$ on $\Omega$, we then find

$$
\sum_{j} Q_{k, \Omega}\left(\psi_{j} u\right)-Q_{k, \Omega}(u)=\frac{1}{k} \int_{\Omega}\left(\sum\left|d \psi_{j}\right|^{2}\right)|u|^{2} \leqslant O\left(\frac{1}{k}\right)|u|^{2} .
$$

By the minimax principle, it follows that the eigenvalues are shifted by at most $O(1 / k)$, thus the asymptotic distribution is not modified as $k \rightarrow+\infty$.

Now, let $x^{0} \in M$ be a given point. We can choose coordinates $\left(x_{1}, \ldots, x_{m}\right)$ centered at $x^{0}$ such that $\left(\partial / \partial x_{1}, \ldots, \partial / \partial x_{m}\right)$ is orthonormal at $x^{0}$ and such that $B=i c(E)$ is "diagonal" at $x^{0}$ :

$$
B\left(x^{0}\right)=\sum_{j=1}^{s} B_{j} d x_{j} \wedge d x_{j+s} ;
$$

here $2 s \leqslant m$ is the rank of the skew-symmetric 2 -form $B\left(x^{0}\right)$, which may depend on $x^{0}$, and $B_{1} \geqslant B_{2} \geqslant \cdots \geqslant B_{s} \geqslant 0$ are its non-zero eigenvalues.

Let us consider the operators obtained by "freezing" the coefficients at $x^{0}$. More specifically, we assume that

- $E$ has constant curvature $B=\sum_{j=1}^{s} B_{j} d x_{j} \wedge d x_{j+s}$. Then there is a local trivialization in which

$$
\begin{aligned}
D_{E} u & =d u-i A \wedge u, \\
A & =\sum_{j=1}^{s} B_{j} x_{j} d x_{j+s} .
\end{aligned}
$$

- $D_{F}$ is flat.

- $\Omega \simeq \mathbb{R}^{n}$ and the metric $g$ is flat : $g=\sum d x_{j} \otimes d x_{j}$. 
- the hermitian form $V$ is constant. We choose an orthonormal frame of $F$ in which $V$ is diagonal, i.e.

$$
\langle V u, u\rangle=\sum_{1 \leqslant \lambda \leqslant r} V_{\lambda}\left|u_{\lambda}\right|^{2}
$$

The connection $D_{k}$ on $E^{k} \otimes F$ can then be written $D_{k} u=d u-i k A \wedge u$ and the quadratic form $Q_{k, \Omega}$ is given by

$$
\begin{aligned}
& Q_{k, \Omega}(u)=\int_{\mathbb{R}^{m}} \frac{1}{k}\left[\sum_{\substack{1 \leqslant j \leqslant s \\
1 \leqslant \lambda \leqslant r}}\left(\left|\frac{\partial u_{\lambda}}{\partial x_{j}}\right|^{2}+\left|\frac{\partial u_{\lambda}}{\partial x_{j+s}}-i k B_{j} x_{j} u_{\lambda}\right|^{2}\right)+\sum_{\substack{j>2 s \\
1 \leqslant \lambda \leqslant r}}\left|\frac{d u_{\lambda}}{d x_{j}}\right|^{2}\right] \\
& -\sum_{1 \leqslant \lambda \leqslant r} V_{\lambda}\left|u_{\lambda}\right|^{2} \text {. }
\end{aligned}
$$

In this situation, $Q_{k, \Omega}$ is a direct sum of quadratic forms acting on each component $u_{\lambda}$ and the computation of $e^{-t \square_{k}}$ is reduced to the following simple cases $(a),(b)$ :

$$
Q(f)=\int_{\mathbb{R}}\left|\frac{d f}{d x}\right|^{2}, \quad \square f=-\frac{d^{2} f}{d x^{2}}
$$

As is well known the heat kernel is given in this case by

$$
\begin{gathered}
K_{t}(x, y)=\frac{1}{\sqrt{4 \pi t}} e^{-(x-y)^{2} / 4 t} . \\
Q(f)=\int_{\mathbb{R}^{2}}\left|\frac{d f}{d x_{1}}\right|^{2}+\left|\frac{d f}{d x_{2}}-i a x_{1} f\right|^{2} .
\end{gathered}
$$

A partial Fourier transform in the $x_{2}$ variable gives

$$
Q(f)=\int_{\mathbb{R}^{2}}\left|\frac{d \hat{f}}{d x_{1}}\left(x_{1}, \xi_{2}\right)\right|^{2}+a^{2}\left(x_{1}-\frac{\xi_{2}}{a}\right)^{2}\left|\hat{f}\left(x_{1}, \xi_{2}\right)\right|^{2}
$$

and the change of variables $x_{1}^{\prime}=x_{1}-x_{2} / a, x_{2}^{\prime}=\xi_{2}$ leads to the so called "harmonic oscillator" energy functional

$$
\begin{aligned}
q(g) & =\int_{\mathbb{R}}\left|\frac{d g}{d x}\right|^{2}+a^{2} x^{2}|g|^{2}, \\
\square & =-\frac{d^{2}}{d x^{2}}+a^{2} x^{2} .
\end{aligned}
$$

The heat kernel of this operator is given by Mehler's formula :

$$
k_{t}(x, y)=\sqrt{\frac{a}{2 \pi \sinh 2 a t}} \exp \left(-\frac{a}{2}(\operatorname{coth} 2 a t)(x-y)^{2}-a(\tanh a t) x y\right) .
$$

One way of obtaining this relation is to observe that the eigenfunctions of $\square$ are

$$
\left(2^{p} p ! \sqrt{\frac{\pi}{a}}\right)^{-1 / 2} \Phi_{p}(\sqrt{a} x), p=0,1,2, \ldots,
$$


with associated eigenvalues $(2 p+1) a$, where $\left(\Phi_{p}\right)$ is the sequence of functions associated to Hermite polynomials :

$$
\Phi_{p}(x)=e^{x^{2} / 2} \frac{d^{p}}{d x^{p}}\left(e^{-x^{2}}\right) .
$$

Therefore we have

$$
k_{t}(x, y)=\sqrt{\frac{a}{\pi}} e^{a\left(x^{2}+y^{2}\right) / 2} \sum_{p=0}^{+\infty} \frac{e^{-(2 p+1) a t}}{2^{p} p ! a^{p}} \frac{d^{p}}{d x^{p}}\left(e^{-a x^{2}}\right) \frac{d^{p}}{d y^{p}}\left(e^{-a y^{2}}\right)
$$

and the summation $\Sigma(x, y)$ can be computed from its Fourier transform

$$
\widehat{\Sigma}(\xi, \eta)=e^{-a t} \exp \left(-\frac{1}{2 a} e^{-2 a t} \xi \eta\right) \cdot \frac{1}{\sqrt{2 a}} e^{-\left(\xi^{2}+\eta^{2}\right) / 4 a} .
$$

The heat kernel operator of $Q$ is thus given by

$$
\left(e^{-t \square} f\right)^{\wedge}\left(x_{1}, \xi_{2}\right)=\int_{\mathbb{R}} k_{t}\left(x_{1}-\frac{\xi_{2}}{a}, y_{1}-\frac{\xi_{2}}{a}\right) \hat{f}\left(y_{1}, \xi_{2}\right) d y_{1} .
$$

By an inverse Fourier transform we obtain the desired heat kernel :

$$
\begin{aligned}
k_{t}\left(x_{1}, x_{2} ; y_{1}, y_{2}\right)+\frac{a}{4 \pi \sinh a t} \exp \left(-\frac{a}{4}(\operatorname{coth} a t)\right. & \left.\left(\left(x_{1}-y_{1}\right)^{2}+\left(x_{2}-y_{2}\right)^{2}\right)\right) \\
& \times \exp \left(\frac{i}{2} a\left(x_{1}+y_{1}\right)\left(x_{2}-y_{2}\right)\right) .
\end{aligned}
$$

The heat kernel associated to a sum of (pairwise commuting) operators $\square_{1}, \ldots, \square_{m}$ acting on disjoint sets of variables is the product of all heat kernels $e^{-t \square_{j}}$. Let $K_{t}^{\lambda}$ be the heat kernel of $Q_{k, \Omega}$ acting on a single component $u_{\lambda}$. The factor in the heat kernel corresponding to the pair of variables $\left(x_{j}, x_{j+s}\right), 1 \leqslant j \leqslant s$ ) is obtained when substituting $k B_{j}$ to $a$ and $t / k$ to $t$. Therefore

$$
\begin{array}{r}
K_{t}^{\lambda}(x, y)=\prod_{j=1}^{s} \frac{k B_{j}}{4 \pi \sinh B_{j} t} \exp \left(-\frac{k B_{j}}{4}\left(\operatorname{coth} B_{j} t\right)\left(\left(x_{2 j-1}-y_{2 j-1}\right)^{2}+\left(x_{2 j}-y_{2 j}\right)^{2}\right)\right. \\
\left.+\frac{i}{2} k B_{j}\left(x_{2 j-1}+y_{2 j-1}\right)\left(x_{2 j}-y_{2 j}\right)\right) \\
\times e^{t V_{\lambda}} \times \frac{1}{(4 \pi t / k)^{m-2 s} / 2} \exp \left(-k \sum_{j>2 s}\left(x_{j}-y_{j}\right)^{2} / 4 t\right) .
\end{array}
$$

On the diagonal of $M$, the global heat kernel $K_{t}$ is thus given by

$$
K_{t}(x, x)=k^{m / 2} \frac{e^{t V}}{(4 \pi)^{m / 2} t^{m / 2-s}} \prod_{j=1}^{s} \frac{B_{j}}{\sinh B_{j} t} .
$$

THEOREm 3.1. - In the variable coefficient case, the heat kernel of $\square_{k}$ admits the asymptotic estimate

$$
K_{t}^{k}(x, x) \sim k^{m / 2} \frac{e^{t V(x)}}{(4 \pi)^{m / 2} t^{m / 2-s}} \prod_{j=1}^{s} \frac{B_{j}(x)}{\sinh B_{j}(x) t}
$$


as $k \rightarrow+\infty$, where $\sim$ is uniform with respect to $x \in M$ and $t$ in a bounded interval $\left.\left[t_{0}, t_{1}\right] \subset\right] 0,+\infty[$.

Proof. - The only thing one has to get convinced of is that $e^{-t \square_{k}}-e^{-t \square_{k}^{0}}$ is $o\left(k^{m / 2}\right)$ at the point $\left(x^{0}, x^{0}\right) \in M \times M$, where $\square_{k}^{0}$ is the operator $\square_{k}$ "freezed" at $x^{0}$. This can be cheecked by means of the well known formula

$$
e^{-t \square_{k}}-e^{-t \square_{k}^{0}}=\int_{0}^{1} e^{-(1-u) t \square_{k}}\left(\square_{k}^{0}-\square_{k}\right) e^{-u t \square_{k}^{0}} d u
$$

once the singularity of $e^{-t \square_{k}}$ along the diagonal is known. We also use the estimate

$$
\square_{k}-\square_{k}^{0}=O\left(\frac{1}{k}\left|x-x^{0}\right| \nabla^{2}+\left(\frac{1}{k}+\left|x-x^{0}\right|^{2}\right) \nabla+\left|x-x^{0}\right|+\frac{1}{k}\right) .
$$

By the localization argument already discussed, we obtain as a consequence the following estimate for the eigenvalues:

Corollary 3.2. - The eigenvalues $\lambda_{j}^{k, \Omega}$ of $Q_{k, \Omega}$ satisfy for every $t>0$ the estimate

$$
\sum_{j=1}^{+\infty} e^{-t \lambda_{j}^{k, \Omega}} \sim k^{m / 2} \int_{\Omega} \frac{\operatorname{tr}\left(e^{t V(x)}\right)}{(4 \pi)^{m / 2} t^{m / 2-s}} \prod_{j=1}^{s} \frac{B_{j}(x)}{\sinh B_{j}(x) t} d \sigma(x) .
$$

This result can be also interpreted in terms of the counting function

$$
N_{k, \Omega}(\lambda)=\#\left\{j ; \lambda_{j}^{k, \Omega} \leqslant \lambda\right\}
$$

and of the spectral density measure (a sum of Dirac measures)

$$
\mu_{k, \Omega}=k^{-m / 2} \frac{d}{d \lambda} N_{k, \Omega}(\lambda) .
$$

In these notations, corollary 3.2 can be restated :

$$
\lim _{k \rightarrow+\infty} \int_{-\infty}^{+\infty} e^{-t \lambda} d \mu_{k, \Omega}(\lambda)=\int_{\Omega} \frac{\operatorname{tr}\left(e^{t V(x)}\right)}{(4 \pi)^{m / 2} t^{m / 2-s}} \prod_{j=1}^{s} \frac{B_{j}(x)}{\sinh B_{j}(x) t} d \sigma(x) .
$$

We thus see that the sequence of measures $\mu_{k, \Omega}$ converges weakly to a measure $\mu_{\Omega}$ whose Laplace transform is given by the right hand side. Inverting the formula, one obtains :

Corollary 3.3. - For almost all $\lambda \in \mathbb{R}$

$$
\left.\left.\lim _{k \rightarrow+\infty} k^{-m / 2} N_{k, \Omega}(\lambda)=\mu_{\Omega}(]-\infty, \lambda\right]\right)=\int_{\Omega} \sum_{j=1}^{r} \nu_{B(x)}\left(V_{j}(x)+\lambda\right) d \sigma(x)
$$

where $\nu_{B(x)}(\lambda)$ is the function on $M \times \mathbb{R}$ defined by

$$
\nu_{B}(\lambda)=\frac{2^{s-m} \pi^{-m / 2}}{\Gamma\left(\frac{m}{2}-s+1\right)} B_{1} \cdots B_{s} \sum_{\left(p_{1}, \ldots, p_{s}\right) \in \mathbb{N}^{s}}\left[\lambda-\Sigma\left(2 p_{j}+1\right) B_{j}\right]_{+}^{\frac{m}{2}-s} .
$$




\section{Proof of the holomorphic Morse inequalities}

Let $X$ be a compact complex manifold, $E$ and $F$ holomorphic hermitian vector bundles of rank 1 and $r$ over $X$. If $X$ is endowed with a hermitian metric $\omega$, Hodge theory shows that the Dolbeault cohomology group $H^{q}\left(X, E^{k} \otimes F\right)$ can be identified with the space of harmonic $(0, q)$-forms with respect to the LaplaceBeltrami operator $\Delta_{k}^{\prime \prime}=\bar{\partial}_{k} \bar{\partial}_{k}^{*}+\bar{\partial}_{k}^{*} \bar{\partial}_{k}$ acting on $E^{k} \otimes F$. We thus have to estimate the zero-eigenspace of $\Delta_{k}^{\prime \prime}$.

In order to apply corollary 3.2 , we first have to compute $\Delta_{k}^{\prime \prime}$ in terms of the hermitian connection $\nabla_{k}$ on $E^{k} \otimes F \otimes \Lambda^{0, q} T^{*} X$ deduced from the Chern connections of $E, F, T X$. What plays now the role of $F$ is the bundle $F \otimes \Lambda^{0, q} T^{*} X$.

The relationship between $\Delta_{k}^{\prime \prime}$ and $\nabla_{k}$ is most easily obtained by means of the Bochner-Kodaira-Nakano identity. In order to simplify the exposition, we assume here that the metric $\omega$ on $X$ is Kähler. For any hermitian holomorphic line bundle $G$ on $X$, the operators $\Delta^{\prime}$ and $\Delta^{\prime \prime}$ of $G$ are related by the B-K-N identity

$$
\Delta^{\prime \prime}=\Delta^{\prime}+[i c(G), \Lambda] .
$$

Here we have $c\left(E^{k} \otimes F\right)=k c(E) \otimes \operatorname{id}_{F}+c(F)$, thus

$$
\Delta_{k}^{\prime \prime}=\Delta_{k}^{\prime}+k[i c(E), \Lambda]+[i c(F), \Lambda] .
$$

At a given point $z^{0} \in X$, we can find a coordinate system $\left(z_{1}, \ldots, z_{n}\right)$ such that $\left(\partial / \partial z_{j}\right)$ is an orthonormal basis of $T X$ and

$$
i c(E)=\frac{i}{2} \sum_{1 \leqslant j \leqslant n} \alpha_{j} d z_{j} \wedge d \bar{z}_{j}
$$

where $\alpha_{1}, \ldots, \alpha_{n}$ are the curvature eigenvalues of $c(E)$ at $z^{0}$. A standard formula gives the expression of the curvature term $[i c(E), \Lambda] u$ for any $(p, q)$-form $u$. With $u=\sum u_{I, J, \lambda} d z_{I} \wedge d \bar{z}_{J} \otimes e_{\lambda}$, we have

$$
\langle[i c(E), \Lambda] u, u\rangle=\sum_{I, J, \lambda}\left(\alpha_{J}-\alpha_{\complement I}\right)\left|u_{I, J, \lambda}\right|^{2}
$$

where $\alpha_{J}=\sum_{j \in J} \alpha_{j}$. In the case of a $(O, q)$-form $u=\sum u_{J, \lambda} d \bar{z}_{J} \otimes e_{\lambda}$ we simply have $\Delta_{k}^{\prime} u=D_{k}^{\prime *} D_{k}^{\prime} u=\nabla_{k}^{\prime *} \nabla_{k}^{\prime} u$ and

$$
\begin{aligned}
\Delta_{k}^{\prime \prime} & =\nabla_{k}^{* *} \nabla_{k}^{\prime}-k V^{\prime}+[i c(F), \Lambda], \\
\left\langle V^{\prime} u, u\right\rangle & =\sum_{J, \lambda} \alpha_{\complement J}\left|u_{J, \lambda}\right|^{2} \quad(\text { here } I=\emptyset) .
\end{aligned}
$$

This is not yet what was needed, since only the $(1,0)$ part $\nabla_{k}^{\prime}$ appears. To get the $(0,1)$ component, we consider $u$ as a $(n, q)$ form with values in $E^{k} \otimes F \otimes \Lambda^{n} T X$. We then get $\Delta_{k}^{\prime} u=D_{k}^{\prime} D_{k}^{\prime *} u$ where

$$
D_{k}^{\prime *} u=-\sum \partial u_{I, J, \lambda} / \partial \bar{z}_{j} d z_{1} \wedge \cdots{\widehat{d z_{j}}}_{\cdots} \wedge d z_{n} \wedge d \bar{z}_{J} \otimes e_{\lambda}
$$

in normal coordinates. Thus $\Delta_{k}^{\prime} u=\nabla_{k}^{\prime \prime *} \nabla_{k}^{\prime \prime} u$ and

$$
\Delta_{k}^{\prime \prime}=\nabla_{k}^{\prime \prime *} \nabla_{k}^{\prime \prime}+k V^{\prime \prime}+\left[i c\left(F \otimes \Lambda^{n} T X\right), \Lambda\right]
$$




$$
\left\langle V^{\prime \prime} u, u\right\rangle=\sum_{J, \lambda} \alpha_{J}\left|u_{J, \lambda}\right|^{2} \quad(\text { here } I=\{1, \ldots, n\}) .
$$

If the metric $\omega$ is non Kähler, we get additional torsion terms, but these terms are independent of $k$. A combination of $\left(4.2^{\prime}\right)$ and $\left(4.2^{\prime \prime}\right)$ yields

$$
\frac{2}{k} \Delta_{k}^{\prime \prime}=\frac{1}{k} \nabla_{k}^{*} \nabla_{k}-V+\frac{1}{k} \Theta
$$

where $\Theta$ is a hermitian form independent of $k$ and

$$
\langle V u, u\rangle=\sum_{J, \lambda}\left(\alpha_{\complement J}-\alpha_{J}\right)\left|u_{J, \lambda}\right|^{2} .
$$

Now apply theorem 3.1 and observe that $\Theta$ does not give any significant contribution to the heat kernel as $k \rightarrow+\infty$. We write here $z_{j}=x_{j}+i y_{j}$, so that

$$
B=i c(E)=\sum_{1 \leqslant j \leqslant n} \alpha_{j} d x_{j} \wedge d y_{j}
$$

The curvature eigenvalues are given by $B_{j}=\left|\alpha_{j}\right|$. We denote $s=s(x)$ the rank of $B(x)$ and order the eigenvalues so that

$$
\left|\alpha_{1}\right| \geqslant \cdots \geqslant\left|\alpha_{s}\right|>0=\alpha_{s+1}=\cdots=\alpha_{n} .
$$

The eigenvalues of $V$ acting on $F \otimes \Lambda^{n} T^{*} X$ are the coefficients $\alpha_{\complement J}-\alpha_{J}$, counted with multiplicity $r$. Therefore

THEOREM 4.4. - The heat kernel associated to $e^{-\frac{2 t}{k} \Delta_{k}^{\prime \prime}}$ in bidegree $(0, q)$ satisfies

$$
K_{t}^{k}(x, x) \sim k^{n} \frac{r \sum_{|J|=q} e^{t\left(\alpha_{\mathrm{C} J}(x)-\alpha_{J}(x)\right)}}{(4 \pi)^{n} t^{n-s}} \prod_{j=1}^{s} \frac{\left|\alpha_{j}(x)\right|}{\sinh \left|\alpha_{j}(x)\right| t}
$$

as $k \rightarrow+\infty$. In particular, if $\lambda_{1}^{k, q} \leqslant \lambda_{2}^{k, q} \leqslant \cdots$ are the eigenvalues of $\frac{1}{k} \Delta_{k}^{\prime \prime}$ in bidegree $(0, q)$, we have

$$
\sum_{j=1}^{+\infty} e^{-2 t \lambda_{j}^{k, q}} \sim r k^{n} \sum_{|J|=q} \int_{X} \frac{e^{t\left(\alpha_{\mathrm{C}_{J}}(x)-\alpha_{J}(x)\right)}}{(4 \pi)^{n} t^{n-s}} \prod_{j=1}^{s} \frac{\left|\alpha_{j}(x)\right|}{\sinh \left|\alpha_{j}(x)\right| t}
$$

for every $t>0$.

At this point, the main idea is to use the eigenspaces in order to construct a finite dimensional subcomplex of the Dolbeault complex with the same cohomology groups. This was already the basic idea in Witten's analytic proof of the standard Morse inequalities. We denote by

$$
\mathcal{H}_{\lambda}^{k, q} \quad, \quad \text { resp. } \mathcal{H}_{\leqslant \lambda}^{k, q}
$$

the $\lambda$-eigenspace of $\frac{1}{k} \Delta_{k}^{\prime \prime}$ acting on $C_{0, q}^{\infty}\left(X, E^{k} \otimes F\right)$, resp. the direct sum of eigenspaces corresponding to all eigenvalues $\leqslant \lambda$. As $\bar{\partial}_{k}$ and $\Delta_{k}^{\prime \prime}$ commute, we see that $\bar{\partial}\left(\mathcal{H}_{\lambda}^{k, q}\right) \subset \mathcal{H}_{\lambda}^{k, q+1}$, thus $\mathcal{H}_{\lambda}^{k, \bullet}$ and $\mathcal{H}_{\leqslant \lambda}^{k, \bullet}$ are finite dimensional subcomplexes of the Dolbeault complex

$$
\bar{\partial}: C_{0, \bullet}^{\infty}\left(X, E^{k} \otimes F\right)
$$


Since $\bar{\partial}_{k} \bar{\partial}_{k}^{*}+\bar{\partial}_{k}^{*} \bar{\partial}_{k}=\Delta_{k}^{\prime \prime}=k \lambda$ id on $\mathcal{H}_{\lambda}^{k, \bullet}$, we see that $\mathcal{H}_{\lambda}^{k, \bullet}$ has trivial cohomology for $\lambda \neq 0$. Since $\mathcal{H}_{0}^{k, \bullet}$ is the space of harmonic forms, we see that $\mathcal{H}_{\leqslant \lambda}^{k, \bullet}$ has the same cohomology as the Dolbeault complex for $\lambda>0$. We will call this complex the Witten $\bar{\partial}$-complex. We need an elementary lemma of linear algebra.

Lemma 4.5. - Set $h_{k}^{q}=\operatorname{dim} H^{q}\left(X, E^{k} \otimes F\right)$. Then for every $t>0$

$$
h_{k}^{q}-h_{k}^{q-1}+\cdots+(-1)^{q} h_{k}^{0} \leqslant \sum_{\ell=0}^{q}(-1)^{q-\ell} \sum_{j=1}^{+\infty} e^{-t \lambda_{j}^{k, \ell}} \text {. }
$$

Proof. - The left hand side is the contribution of the 0 eigenvalues in the right hand side. All we have to check is that the contribution of the other eigenvalues is $\geqslant 0$. The contribution of the eigenvalues such that $\lambda_{j}^{k, \ell}=\lambda>0$ is

$$
e^{-t \lambda} \sum_{\ell=0}^{q}(-1)^{q-\ell} \operatorname{dim} \mathcal{H}_{\lambda}^{k, \ell}
$$

As $\mathcal{H}_{\lambda}^{k, \bullet}$ is exact, one easily sees that the last sum is equal to the dimension of $\bar{\partial} \mathcal{H}_{\lambda}^{k, q} \subset \mathcal{H}_{\lambda}^{k, q+1}$, hence $\geqslant 0$.

Combining theorem 4.4 with lemma 4.5, we get

$$
\begin{aligned}
& h_{k}^{q}-h_{k}^{q-1}+\cdots+(-1)^{q} h_{k}^{0} \leqslant o\left(k^{n}\right)+ \\
& r k^{n} \sum_{\ell=0}^{q}(-1)^{q-\ell} \sum_{|J|=\ell} \int_{X} \frac{\prod_{j \leqslant s}\left|\alpha_{j}\right| \cdot e^{t\left(\alpha_{\left.\mathrm{C}_{J}-\alpha_{J}-\sum\left|\alpha_{j}\right|\right)}\right.}}{2^{2 n-s} \pi^{n} t^{n-s} \prod_{j \leqslant s}\left(1-e^{-2 t\left|\alpha_{j}\right|}\right)} .
\end{aligned}
$$

This inequality is valid for any $t>0$, so we can let $t$ tend to $+\infty$. It is clear that $\alpha_{\complement}-\alpha_{J}-\sum\left|\alpha_{j}\right|$ is always $\leqslant 0$, thus the integrand tends to 0 at every point where $s<n$. When $s=n$, we have $\left.\alpha_{\complement J}(x)-\alpha_{J} x\right)-\sum\left|\alpha_{j}(x)\right|=0$ if and only if $\alpha_{j}(x)>0$ for every $j \in \complement J$ and $\alpha_{j}(x)<0$ for every $j \in J$. This implies $x \in X(\ell, E)$; in this case there is only one multi-index $J$ satisfying the above conditions and the limit is $(2 \pi)^{-n}\left|\alpha_{1} \cdots \alpha_{n}\right|$. By the monotone convergence theorem, our sum of integrals converges to

$$
\sum_{\ell=0}^{q}(-1)^{q-\ell} \int_{X(\ell, E)}(2 \pi)^{-n}\left|\alpha_{1} \cdots \alpha_{n}\right| d \sigma=\frac{1}{n !} \int_{X(\leqslant q, E)}(-1)^{q}\left(\frac{i}{2 \pi} c(E)\right)^{n} .
$$

\section{Applications to algebraic geometry}

Let $E$ be a holomorphic line bundle over a compact connected complex manifold $X$ of dimension $n$ and $V_{k}=H^{0}\left(X, E^{k}\right)$. If $Z\left(V_{k}\right)$ denotes the set of common zeroes of all sections in $V_{k}$, there is a natural holomorphic map

$$
\Phi_{k}: X \backslash Z\left(V_{k}\right) \longrightarrow \mathbb{P}\left(V_{k}^{*}\right)
$$


which sends a point $x \in X \backslash Z\left(V_{k}\right)$ to the hyperplane $H \subset V_{k}$ of sections $\sigma \in V_{k}$ such that $\sigma(x)=0$.

When $E$ is $>0$, one can construct many sections of high tensor powers $E^{k}$ (e.g. by Hörmander's $L^{2}$ estimates). For $k \geqslant k_{0}$ large enough, the "base locus" $Z\left(V_{k}\right)$ is empty, the sections in $V_{k}$ separate any two points of $X$ and generate all 1-jets at any point. Then $\Phi_{k}$ gives an embedding of $X$ in some projective space $\mathbb{P}^{N}$.This gives the famous Kodaira embedding theorem : a compact complex manifold $X$ is projective algebraic if and only if $X$ has a hermitian line bundle $E$ with positive curvature.

The Grauert-Riemenschneider conjecture $[\mathrm{G}-\mathrm{R}]$ was an attempt to characterize the more general class of Moishezon varieties in terms of semi-positive line bundles. Let us first recall a few definitions. The algebraic dimension $a(X)$ is the transcendence degree of the field $\mathcal{M}(X)$ of meromorphic functions on $X$. A wellknown theorem of Siegel asserts that $0 \leqslant a(X) \leqslant n$. A manifold (or variety) $X$ is said to be Moishezon if $a(X)=n$.

Grauert-Riemenschneider Conjecture (1970). — A compact complex variety $Y$ is Moishezon if and only if there is a proper non singular modification $X \rightarrow Y$ and a line bundle $E$ over $X$ such that the curvature is $>0$ on a dense open subset.

When $Y$ is Moishezon, it is well known that there exists a projective algebraic modification $X$; therefore $E$ can even be taken $>0$ everywhere on such an $X$. Siu [Siu 2] solved the conjecture by proving the converse statement in 1984; he even showed that $X$ is Moishezon as soon as $i c(E) \geqslant 0$ everywhere and $i c(E)>0$ in at least one point. We will see that Morse inequalities give a still stronger criterion, requiring only the positivity of some curvature integral.

Since $\mathcal{M}(Y) \simeq \mathcal{M}(X)$, we only have to show that $X$ itself is Moishezon. This will be done by producing many sections of $E^{k}$. For $x=1$, the strong Morse inequality (1.4) gives

$$
h^{1}\left(E^{k}\right)-h^{0}\left(E^{k}\right) \leqslant-\frac{k^{n}}{n !} \int_{X(\leqslant 1, E)}\left(\frac{i}{2 \pi} c(E)\right)^{n}+o\left(k^{n}\right) .
$$

In particular, we get the lower bound

$$
h^{0}\left(E^{k}\right) \geqslant \frac{k^{n}}{(2 \pi)^{n} n !} \int_{X(\leqslant 1, E)}(i c(E))^{n}-o\left(k^{n}\right) .
$$

By definition, the Kodaira dimension $\kappa(E)$ is the supremum of the dimension of the images $Y_{k}=\Phi_{k}\left(X \backslash Z\left(V_{k}\right)\right) \subset \mathbb{P}\left(V_{k}^{*}\right)$ for all integers $k>0$. Since the field of meromorphic functions on $X$ obtained by restriction of rational functions of $\mathbb{P}\left(V_{k}^{*}\right)$ to $Y_{k}$ has transcendence degree $\operatorname{dim} Y_{k}$, we infer that $\kappa(E)=\sup \operatorname{dim} Y_{k} \leqslant a(X)$. The following elementary lemma is needed.

Lemma 5.2. - For every line bundle $E$, there is a constant $C>0$ such that

$$
\operatorname{dim} H^{0}\left(X, E^{k}\right) \leqslant C k^{\kappa(E)} .
$$


The proof proceeds as follows : select a hermitian metric on $E$ and a family of balls $B_{j}=B\left(z_{j}, r_{j}\right) \subset B_{j}^{\prime}=B\left(z_{j}, 2 r_{j}\right)$ covering $X$, on which $E$ is trivial. If $E^{k}$ had too many sections, one could solve a linear system in many unknowns to get a section $s$ vanishing at a high order $m$ at all centers $z_{j}$. Then Schwarz'lemma gives

$$
\|s\|_{\infty}=\sup \|s\|_{B_{j}} \leqslant 2^{-m} C^{k} \sup \|s\|_{B_{j}^{\prime}} \leqslant 2^{-m} C^{k}\|s\|
$$

where $C$ is the oscillation of the metric on $B_{j}^{\prime}$. Thus $m \leqslant k \log C / \log 2$ if $s \neq 0$. Since the sections of $E^{k}$ are essentially constant along the fibers of $\Phi_{k}$, only $m^{\operatorname{dim} Y_{k}} \#\left\{z_{j}\right\}$ equations are needed to make $s$ vanish at order $m$. Therefore we can choose $m \simeq C_{1} h^{0}\left(E^{k}\right)^{1 / \operatorname{dim} Y_{k}}$, so that

$$
h^{0}\left(E^{k}\right) \leqslant C_{2} m^{\operatorname{dim} Y_{k}} \leqslant C_{3} k^{\kappa(E)} .
$$

Combining (5.1) and lemma 5.2, we get the following result which implies the Grauert-Riemenschneider conjecture.

TheOREM 5.3. - If a hermitian line bundle $E$ verifies the integral condition $\int_{X(\leqslant 1, E)}(i c(E))^{n}>0$, then $\kappa(E)=n$, in particular $X$ is Moishezon.

Another application of the heat kernel estimates is a generalization of G. Kempf's distortion inequalities ([Kem], [Ji]) to all projective algebraic manifolds.

Let $E$ be a positive hermitian line bundle over a projective manifold $X$, equipped with a hermitian metric $\omega$. Then $V_{k}=H^{0}\left(X, E^{k}\right)$ has a natural hermitian metric given by the global $L^{2}$ norm of sections. For $k \geqslant k_{0}$ large enough, $\Phi_{k}$ is an embedding and $E^{k}$ can be identified to the pull-back $\Phi_{k}^{*} O(1)$. We want to compare the original metric || of $E$ and the metric ||$_{F S}$ induced by the Fubini-Study metric of $O(1)$.

Let $\left(s_{1}, \ldots, s_{N}\right)$ be an orthonormal basis of $H^{0}\left(X, E^{k}\right)$. It is not difficult to check that

$$
|\xi|_{F S}^{2}=\frac{|\xi|^{2}}{\left|s_{1}(x)\right|^{2}+\cdots+\left|s_{N}(x)\right|^{2}} \text { for } \xi \in E_{x}^{k}
$$

thus all that we need is to get an estimate of $\sum\left|s_{j}(x)\right|^{2}$. However, this sum is the contribution of the 0 eigenvalue in the heat kernel

$$
K_{t}^{k}(x, x)=\sum_{j=1}^{+\infty} e^{-2 t \lambda_{j}^{k}}\left|\psi_{j}(x)\right|^{2}
$$

associated to $\frac{2}{k} \square_{k}^{\prime \prime}$ in bidegree $(0,0)$. We observe that non zero eigenvalues $\lambda_{j}^{k}$ are also eigenvalues in bidegree $(0,1)$, since $\bar{\partial}$ is injective on the corresponding eigenspaces. The associated eigenfunctions are $\bar{\partial} \psi_{j} / \sqrt{k \lambda_{j}^{k}}$, for

$$
\left\|\bar{\partial} \psi_{j}\right\|^{2}=\left\langle\Delta_{k}^{\prime \prime} \psi_{j}, \psi_{j}\right\rangle=k \lambda_{j}^{k}
$$

Thus the summation

$$
\sum_{j=1}^{+\infty} e^{-2 t \lambda_{j}^{k}}\left|\bar{\partial} \psi_{j}(x)\right|^{2}
$$


is bounded by the heat kernel in bidegree $(0,1)$, which is itself bounded by $k^{n} e^{-c t}$ with $c>0$ (note that $\alpha_{\complement J}-\alpha_{J}-\sum\left|\alpha_{j}\right|<0$ on $X$ for $|J|=1$ ). Taking $t=k^{\varepsilon}$ with $\varepsilon$ small, one can check that all estimates remain uniformly valid and that the contribution of the non zero eigenfunctions in $K_{t}^{k}(x, x)$ becomes negligible in $C^{0}$ norm. Then theorem 4.4 shows that

$$
\sum\left|s_{j}(x)\right|^{2} \sim K_{t}^{k}(x, x) \sim k^{n}(2 \pi)^{-n}\left|\alpha_{1}(x) \cdots \alpha_{n}(x)\right|
$$

as $t=k^{\varepsilon} \rightarrow+\infty$. For $\xi \in E_{x}^{k}$ we get therefore the $C^{0}$ convergence

$$
\frac{|\xi|^{2}}{|\xi|_{F S}^{2}} \sim\left(\frac{k}{2 \pi}\right)^{n}\left|\alpha_{1}(x) \cdots \alpha_{n}(x)\right| \quad \text { as } k \rightarrow+\infty .
$$

As a consequence, the Fubini-Study metric on $E$ induced by $\Phi_{k}$ converges to the original metric. G. Tian [Ti] has proved that this last convergence statement holds in fact in norm $C^{4}$.

\section{The case of $q$-convex manifolds}

Thierry Bouche [Bou 1] has obtained an extension of the holomorphic Morse inequalities to the case of strongly $q$-convex manifolds. We explain here the main ideas used.

A complex (non compact) manifold $X$ of dimension $n$ is strongly $q$-convex in the sense of Andreotti and Grauert [A-G] if there exists a $C^{\infty}$ exhaustion function $\psi$ on $X$ such that $i \partial \bar{\partial} \psi$ has at least $n-q+1$ positive eigenvalues outside a compact subset of $X$. In this case, the Andreotti-Grauert theorem shows that all cohomology groups $H^{m}(X, \mathcal{F})$ with values in a coherent analytic sheaf are finite dimensional for $m \geqslant q$.

Theorem 6.1. - Let E, $F$ be holomorphic vector bundles over $X$ with rank $E=1$, rand $F=r$. Assume that $X$ is strongly $q$-convex and that $E$ has a metric for which $i c(E)$ has at least $n-p+1$ nonnegative eigenvalues outside a compact subset. Then for all $m \geqslant p+q-1$ the following strong Morse inequalities hold :

$$
\sum_{\ell=m}^{n}(-1)^{\ell-m} \operatorname{dim} H^{\ell}\left(X, E^{k} \otimes F\right) \leqslant r \frac{k^{n}}{n !} \int_{X(\geqslant m, E)}(-1)^{m}\left(\frac{i}{2 \pi} c(E)\right)^{n}+o\left(k^{n}\right) .
$$

Proof. - For every $c \in \mathbb{R}$, we consider the sublevel sets

$$
X_{c}=\{x \in X ; \psi(x)<c\} .
$$

Select $c_{0}$ such that $i \partial \bar{\partial} \psi$ has $n-q+1$ positive eigenvalues on $X \backslash X_{c}$. One can choose a hermitian metric $\omega_{0}$ on $X$ in such a way that the eigenvalues $\gamma_{1}^{0} \leqslant \cdots \leqslant \gamma_{n}^{0}$ of $i \partial \bar{\partial} \psi$ with respect to $\omega_{0}$ satisfy

$$
-\frac{1}{n} \leqslant \gamma_{1}^{0} \leqslant \cdots \leqslant \gamma_{q-1}^{0} \leqslant 1 \quad \text { and } \quad \gamma_{q}^{0}=\cdots=\gamma_{n}^{0}=1 \text { on } X \backslash X_{c_{0}} ;
$$


this can be achieved by taking $\omega_{0}$ equal to $i \partial \bar{\partial} \psi$ on a $C^{\infty}$ subbundle of $T X$ of rank $n-q+1$ on which $i \partial \bar{\partial} \psi$ is positive, and $\omega_{0}$ very large on the orthogonal complement. We set $\omega=e^{\rho} \omega_{0}$ where $\rho$ is a function increasing so fast at infinity that $\omega$ will be complete.

More important, we multiply the metric of $E$ by a weight $e^{-\chi \circ \psi}$ where $\chi$ is a convex increasing function. The resulting hermitian line bundle is denoted $E_{\chi}$. For any $(0, m)$ form $u$ with values in $E^{k} \otimes F$, viewed as an $(n, m)$ form with values in $E^{k} \otimes F \otimes \Lambda^{n} T X$, the Bochner-Kodaira-Nakano formula implies an inequality

$$
\left\langle\Delta_{k}^{\prime \prime} u, u\right\rangle \geqslant \int_{X} k\left\langle\left[i c\left(E_{\chi}\right), \Lambda\right] u, u\right\rangle+\langle\Theta u, u\rangle
$$

where $\Theta$ depends only on the curvature of $F \otimes \Lambda^{n} T X$ and the torsion of $\omega$. By the formulas of $\S 4$, we have

$$
\left\langle\left[i c\left(E_{\chi}\right), \Lambda\right] u, u\right\rangle \geqslant\left(\alpha_{1}+\cdots+\alpha_{m}\right)|u|^{2}
$$

where $\alpha_{1} \leqslant \cdots \leqslant \alpha_{n}$ are the eigenvalues of

$$
i c\left(E_{\chi}\right)=i c(E)+i \partial \bar{\partial}(\chi \circ \psi) \geqslant i c(E)+\left(\chi^{\prime} \circ \psi\right) i \partial \bar{\partial} \psi \text {. }
$$

If $\beta$ is the lowest eigenvalue of $i c(E)$ with respect to $\omega$, we find

$$
\begin{aligned}
\alpha_{j} & \geqslant \beta+\left(\chi^{\prime} \circ \psi\right) \gamma_{j}^{0} / e^{\rho}, \\
\alpha_{1}+\cdots+\alpha_{m} & \geqslant m \beta+\left(\chi^{\prime} \circ \psi\right)\left(\gamma_{1}^{0}+\cdots+\gamma_{m}^{0}\right) / e^{\rho},
\end{aligned}
$$

and by (6.2) we get for all $m \geqslant q$ :

$$
\alpha_{1}+\cdots+\alpha_{m} \geqslant m \beta+\frac{1}{n} e^{-\rho} \chi^{\prime} \circ \psi \text { on } X \backslash X_{c_{0}}
$$

It follows that one can choose $\chi$ increasing very fast in such a way that the Bochner inequality becomes

$$
\left\langle\Delta_{k}^{\prime \prime} u, u\right\rangle \geqslant k \int_{X \backslash C_{c_{0}}} A(x)|u(x)|^{2}-C \int_{X}|u(x)|^{2}
$$

where $A \geqslant 1$ is a function tending to $+\infty$ at infinity on $X$ and $C \geqslant 0$. Now, Rellich's lemma easily shows that $\Delta_{k}^{\prime \prime}$ has a compact resolvent. Hence the spectrum of $\Delta_{k}^{\prime \prime}$ is discrete and its eigenspaces are finite dimensional. Standard arguments also show the following :

Lemma 6.4. - When $\chi$ increases sufficiently fast at infinity, the space $\mathcal{H}^{m}\left(X, E_{\chi}^{k} \otimes F\right)$ of $L^{2}$-harmonic forms of bidegree $(0, m)$ for $\Delta_{k}^{\prime \prime}$ is isomorphic to the cohomology group $H^{m}\left(X, E^{k} \otimes F\right)$ for all $k \in \mathbb{N}$ and $m \geqslant q$.

For a domain $\Omega \subset \subset X$, we consider the quadratic form

$$
Q_{\Omega}^{k, m}(u)=\frac{1}{k} \int_{\Omega}\left|\bar{\partial}_{k} u\right|^{2}+\left|\bar{\partial}_{k}^{*} u\right|^{2}
$$

with Dirichlet boundary conditions on $\partial \Omega$. We denote by $\mathcal{H}_{\leqslant \lambda, \Omega}^{k, m}$ the direct sum of all eigenspaces of $Q_{\Omega}^{k, m}$ corresponding to eigenvalues $\leqslant \lambda\left(\right.$ i.e. $\leqslant k \lambda$ for $\Delta_{k}^{\prime \prime}$ ). 
Lemma 6.5. - For every $\lambda \geqslant 0$ and $\varepsilon>0$, there exists a domain $\Omega \subset \subset X$ and an integer $k_{0}$ such that

$$
\operatorname{dim} \mathcal{H}_{\leqslant \lambda, \Omega}^{k, m} \leqslant \operatorname{dim} \mathcal{H}_{\leqslant \lambda, X}^{k, m} \leqslant \operatorname{dim} \mathcal{H}_{\leqslant \lambda+\varepsilon, \Omega}^{k, m} \text { for } k \geqslant k_{0} .
$$

Proof. - The left hand inequality is a straightforward consequence of the minimax principle, because the domain of the global quadratic form $Q_{\Omega}^{k, m}$ is contained in the domain of $Q_{X}^{k, m}$.

For the other inequality, let $u \in \mathcal{H}_{\leqslant \lambda, X}^{k, m}$. Then (6.3) gives

$$
k \int_{X \backslash X_{c_{0}}} A|u|^{2}-C \int_{X_{c_{0}}}|u|^{2} \leqslant k \lambda \int_{X}|u|^{2} .
$$

Choose $c_{2}>c_{1}>c_{0}$ so that $A(x) \geqslant a$ on $X \backslash X_{c_{1}}$ and a cut-off function $\varphi$ with compact support in $X_{c_{2}}$ such that $0 \leqslant \varphi \leqslant 1$ and $\varphi=1$ on $X_{c_{1}}$. Then we find

$$
\int_{X \backslash X_{c_{1}}}|u|^{2} \leqslant \frac{C+k \lambda}{k a} \int_{X}|u|^{2} .
$$

For a large enough, we get $\int_{X \backslash X_{c_{1}}}|u|^{2} \leqslant \varepsilon\|u\|^{2}$. Set $\Omega=X_{c_{2}}$. Then

$$
\begin{aligned}
Q_{\Omega}^{k, m}(\varphi u) & \left.=\frac{1}{k} \int_{\Omega}\left|\bar{\partial} \varphi \wedge u+\varphi \bar{\partial}_{k} u\right|^{2}+\mid \varphi \bar{\partial}_{k}^{*} u-\partial \varphi\right\lrcorner\left. u\right|^{2} \\
& \leqslant(1+\varepsilon) Q_{X}^{k, m}(u)+\frac{C^{\prime}}{k}\left(1+\frac{1}{\varepsilon}\right)\|u\|^{2} \\
& \leqslant(1+\varepsilon)\left(\lambda+\frac{C^{\prime}}{k \varepsilon}\right)\|u\|^{2} .
\end{aligned}
$$

As $\|\varphi u\|^{2} \geqslant \int_{X_{c_{1}}}|u|^{2} \geqslant(1-\varepsilon)\|u\|^{2}$, we infer

$$
Q_{\Omega}^{k, m}(\varphi u) \leqslant \frac{1+\varepsilon}{1-\varepsilon}\left(\lambda+\frac{C^{\prime}}{k \varepsilon}\right)\|\varphi u\|^{2} .
$$

If $\varepsilon$ is replaced by a suitable smaller number and $k$ taken large enough, we obtain $Q_{\Omega}^{k, m}(v) \leqslant(\lambda+\varepsilon)\|v\|^{2}$ for all $v \in \varphi \mathcal{H}_{\leqslant \lambda, X}^{k, m}$. Then the right hand inequality in lemma 6.5 follows by the minimax principle.

Now, corollary 3.3 easily computes the counting function $N_{\Omega}^{k, m}$ for the eigenvalues :

$$
\lim _{\lambda \rightarrow 0_{+}} \lim _{k \rightarrow+\infty} k^{-n} N_{\Omega}^{k, m}(\lambda)=\frac{r}{n !} \int_{X\left(m, E_{\chi}\right)}(-1)^{m}\left(\frac{i}{2 \pi} c\left(E_{\chi}\right)\right)^{n} .
$$

Applying this to the Witten complex $\mathcal{H}_{\leqslant \lambda, X}^{k, \bullet}$, we easily infer the inequality of theorem 6.1, except that $c(E)$ is replaced by $c\left(E_{\chi}\right)$. However, up to now, the inequality is valid for all $m \geqslant q$. Take the convex function $\chi$ equal to 0 on $\left.]-\infty, c_{0}\right]$. Then

$$
i c\left(E_{\chi}\right)=i c(E)+i \partial \bar{\partial}(\chi \circ \psi)
$$


coincides with $i c(E)$ on $X_{c_{0}}$ and has at most $(p-1)+(q-1)$ negative eigenvalues on $X \backslash X_{c_{0}}$. Hence $X\left(m, E_{\chi}\right)=X(m, E)$ for $m \geqslant p+q-1$ and $i c\left(E_{\chi}\right)=i c(E)$ on these sets. Theorem 6.1 is proved.

As a corollary, one obtains a general a priori estimate for the Monge-Ampère operator $(i \partial \bar{\partial})^{n}$ on $q$-convex manifolds.

Corollary 6.6. - Let $X$ be a strongly $q$-convex manifold and $\varphi$ a $C^{\infty}$ function on $X$, weakly $p$-convex outside a compact subset of $X$. For $\ell=0,1, \ldots, n$, let $G_{\ell}$ be the open set of points where $i \partial \bar{\partial} \varphi$ is non degenerate and admits $\ell$ negative eigenvalues. Then for all $m \geqslant p=q-1$

$$
\sum_{\ell=m}^{n} \int_{G_{\ell}}(i \partial \bar{\partial} \varphi)^{m} \text { has the sign of }(-1)^{m} \text {. }
$$

This result has been first obtained by Y.T. Siu [Siu 4] for $q$-convex domains in a Stein manifold. At that time, the $q$-convex case of the inequalities was not yet available and Siu had to rely on a rather sophisticated approximation argument of Stein manifolds by algebraic varieties; the proof could then be reduced to the compact case.

The general statement given above is in fact a direct consequence of theorem 6.1 : take for $E$ the trivial bundle $E=\mathcal{O}_{X}$ equipped with the metric defined by the weight $e^{-\varphi}$ and $F=\mathcal{O}_{X}$. Since $H^{m}\left(X, E^{k}\right)=H^{m}\left(X, \mathcal{O}_{X}\right)$ is independent of $k$ and finite dimensional, theorem 6.1 implies

$$
k^{n} \sum_{\ell=m}^{n} \int_{G_{\ell}}(-1)^{m}(i \partial \bar{\partial} \varphi)^{n} \geqslant \text { constant }-o\left(k^{n}\right)
$$

for all $k \geqslant k_{0}$ and $m \geqslant p+q-1$, whence the result.

\section{Holomorphic Morse inequalities for vector bundles}

A natural question arising in connection with our Morse inequalities is whether one can extend the inequalities for high tensor powers of a vector bundle $E$ of rank $\geqslant 2$. Since $E^{\otimes k}$ is decomposable for $k \geqslant 2$ (e.g. $E^{\otimes 2}=S^{2} E \oplus \Lambda^{2} E$ ) we are led to consider only irreducible tensor powers of $E$, i.e. the irreducible representations of the linear group $G \ell(E)$. This is done by Getzler [Ge 2], in the general framework of Lie group theory and representations. As we are only dealing with the case of the full linear group, we will give here an elementary presentation. We first recall some ideas from Borel-Weil's theory and a special case of Bott's formula $[$ Bot $]$.

Let $V$ be a complex vector space of dimension $r$ and $M(V)$ the flag manifold of $V$, i.e. the set of all $(r+1)$-tuples $z=\left(V_{0}, V_{1}, \ldots, V_{r}\right)$ with $V=V_{0} \supset V_{1} \supset$ 
$\cdots \supset V_{r}=\{0\}$ and $\operatorname{codim} V_{j}=j$. On $M(V)$ we have canonical line bundles $Q_{j}$ such that

$$
Q_{j, z}=V_{j-1} / V_{j}, 1 \leqslant j \leqslant r
$$

For any $r$-tuple $\left(a_{1}, \ldots, a_{r}\right) \in \mathbb{Z}^{r}$, we set

$$
Q^{a}=Q_{1}^{a_{1}} \otimes \cdots \otimes Q_{r}^{a_{r}} .
$$

As $G \ell(V)$ acts equivariantly on $Q^{a} \rightarrow M(V)$, the spaces of sections

$$
\Gamma^{a} V=H^{0}\left(M(V), Q^{a}\right)
$$

are equipped with a natural $G \ell(V)$ action. Observe that $Q^{(1, \ldots, 1)}$ is isomorphic to the trivial bundle $M(V) \times \operatorname{det} V$ (but of course the action of $G \ell(V)$ on $\operatorname{det} V$ is non trivial). To describe $\Gamma^{a} V$, we can therefore assume that all $a_{j}$ are nonnegative. Then any section $\sigma \in \Gamma^{a} V$ can be viewed as a polynomial $P_{\sigma}\left(\xi_{1}, \ldots, \xi_{r}\right)$ on $\left(V^{*}\right)^{r}$ as follows : if $\xi_{1}, \ldots, \xi_{r} \in V^{*}$ are linearly independent, one can associate to $\left(\xi_{j}\right)$ the flag $z=\left(V_{j}\right)$ defined by $V_{j}=\xi_{1}^{-1}(0) \cap \cdots \cap \xi_{j}^{-1}(0)$. Then $\xi_{j}$ induces a well defined linear form $\tilde{\xi}_{j}$ on $Q_{j, z}=V_{j-1} / V_{j}$ and we set

$$
P_{\sigma}\left(\xi_{1}, \ldots, \xi_{r}\right)=\left(\tilde{\xi}_{1}^{a_{1}} \otimes \cdots \otimes \tilde{\xi}_{r}^{a_{r}}\right) \cdot \sigma(z) .
$$

It is clear that $P_{\sigma}$ remains locally bounded on a neighborhood of the hypersurface $\operatorname{det}\left(\xi_{1}, \ldots, \xi_{r}\right)=0$; therefore $P_{\sigma}$ extends to a polynomial on $\left(V^{*}\right)^{r}$ that is homogeneous of degree $a_{j}$ in the variable $\xi_{j}$. Also, neither the flag $z$ nor the linear forms $\tilde{\xi}_{j}$ are modified if we replace $\xi_{j}$ by $\xi_{j}+\sum_{k<j} \lambda_{j k} \xi_{k}$. It follows that $P_{\sigma}$ satisfies the relation

$$
P_{\sigma}\left(\xi_{j}+\sum_{k<j} \lambda_{j k} \xi_{k}\right)=P_{\sigma}\left(\xi_{1}, \ldots, \xi_{r}\right), \forall \lambda_{j k} \in \mathbb{C}
$$

and conversely any polynomial $P$ of multidegree $\left(a_{1}, \ldots, a_{r}\right)$ satisfying this condition yields a (unique) section $\sigma \in \Gamma^{a} V$. Hence $\Gamma^{a} V$ is the subspace of tensors in $S^{a_{1}} V \otimes \cdots \otimes S^{a_{r}} V$ enjoying the above additional antisymmetry properties. In particular we have

$$
\begin{aligned}
S^{k} V & =\Gamma^{(k, 0, \ldots, 0)} V, \\
\Lambda^{k} V & =\Gamma^{(1, \ldots, 1,0, \ldots, 0)} V, \quad(k \text { first integers }=1) .
\end{aligned}
$$

We will see soon that $\Gamma^{a} V=\{0\}$ unless $a_{1} \geqslant a_{2} \geqslant \cdots \geqslant a_{r}$. The spaces $\Gamma^{a} V\left(a_{1} \geqslant \cdots \geqslant a_{r}\right)$ can be seen to be irreducible representations of $G \ell(V)$. As is well known in representation theory, $\left(\Gamma^{a} V\right)$ is in fact the complete list of irreducible representations of $G \ell(V)$ up to isomorphism.

Assume now that $V$ is equipped with a hermitian metric. Then any flag $z^{0} \in M(V)$ is represented by an orthonormal basis $\left(e_{1}, \ldots, e_{r}\right)$ such that $V_{j}^{0}=$ $\operatorname{Vect}\left(e_{j+1}, \ldots, e_{r}\right)$. Now $z^{0}$ is contained in the affine chart of points $z=\left(V_{j}\right)$ with

$$
V_{j}=\operatorname{Vect}\left(v_{j+1}, \ldots, v_{r}\right), v_{k}=e_{k}+\sum_{j<k} z_{j k} e_{j}
$$


where $\left(z_{j k}\right) \in \mathbb{C}^{n(n-1) / 2}$ are the affine coordinates of $z$. The canonical metric on $Q^{a}$ induced by $V$ has curvature

$$
c\left(Q^{a}\right)_{z^{0}}=\sum_{1 \leqslant j<j \leqslant n}\left(a_{j}-a_{k}\right) d z_{j k} \wedge d \bar{z}_{j k}
$$

we will omit the easy (and standard) computation. By homogeneity, we see that $Q^{a}$ is positive as soon as $a_{1}>a_{2}>\cdots>a_{r}$. On the other hand, when $a_{j-1}<a_{j}$, we see that $Q^{a}$ is negative along the $\mathbb{P}^{1}$ line in $M(V)$ obtained by fixing all $V_{k}=V_{k}^{0}$ except $V_{j}$. Therefore $\Gamma^{a} V=H^{0}\left(M(V), Q^{a}\right)=\{0\}$ in this case.

Assume from now on that $a_{1} \geqslant \cdots \geqslant a_{r}$, and more specifically that

$$
a_{1}=\cdots=a_{s_{1}}>a_{s_{1}+1}=\cdots=a_{s_{2}}>\cdots>a_{s_{m-1}+1}=\cdots=a_{s_{m}},
$$

where $s_{m}=r$. As $Q_{j+1, z} \otimes \cdots \otimes Q_{k, z} \simeq \operatorname{det}\left(V_{j} / V_{k}\right)$, we see that $Q^{a}$ is the pull back of the bundle

$$
Q_{s}^{a}=\operatorname{det}\left(V / V_{s_{1}}\right)^{a_{s_{1}}} \otimes \cdots \otimes \operatorname{det}\left(V_{s_{m-1}} / V_{s_{m}}\right)^{a_{s_{m}}}
$$

over the manifold $M_{s}(V)$ of partial flags

$$
V \supset V_{s_{1}} \supset \cdots \supset V_{s_{m}}=\{0\}
$$

via the obvious projection $\pi_{s}: M(V) \rightarrow M_{s}(V)$. On $M_{s}(V)$ we have a formula completely analogous to $(7.2)$, where the only indices $(j, k)$ involved are those for which $a_{j}>a_{k}$. Thus $Q_{s}^{a}$ is ample and $Q^{a}=\pi_{s}^{\star} Q_{s}^{a}$, in particular

$$
H^{0}\left(M_{s}(V), Q_{s}^{a}\right)=H^{0}\left(M(V), Q^{a}\right)=\Gamma^{a} V .
$$

Now let $E, F$ be holomorphic vector bundles over a compact manifold $X$ and let

$$
n=\operatorname{dim}_{\mathbb{C}} X, r=\operatorname{rank} E, r^{\prime}=\operatorname{rank} F .
$$

We want to get asymptotic estimates for the dimension of cohomology groups $H^{q}\left(X, \Gamma^{k a} E \otimes F\right)$ as $k \rightarrow+\infty$. For that, we introduce the flag bundle

$$
M_{s}(E) \rightarrow X,
$$

where $s=\left(s_{1}, \ldots, s_{m}\right)$ is defined as above, and we consider the universal line bundle $Q_{s}^{a}$ over $M_{s}(E)$. As $Q_{s}^{a}$ is ample along the fibers of $\pi_{s}: M_{s}(E) \rightarrow X$, the higher direct images

$$
R^{q}\left(\pi_{s}\right)_{\star}\left(Q_{s}^{k a} \otimes \pi_{s}^{\star} F\right), \quad q \geqslant 1
$$

vanish for $k \geqslant k_{0}$. By (7.3) we get

$$
\left(\pi_{s}\right)_{\star}\left(Q_{s}^{k a} \otimes \pi_{s}^{\star} F\right)=\Gamma^{k a} E \otimes F .
$$

The Leray spectral sequence gives the isomorphism

$$
H^{q}\left(X, \Gamma^{k a} E \otimes F\right) \simeq H^{q}\left(Y, Q_{s}^{k a} \otimes \pi_{s}^{\star} F\right), \quad Y=M_{s}(E),
$$

and we are reduced to applying Morse inequalities to tensor powers of the line bundle $Q_{s}^{a}$. We still need a formula for the curvature of $Q_{s}^{a}$ with the metric induced by a given hermitian metric on $E$. Let $z^{0} \in M_{s}\left(E_{x^{0}}\right)$ be a point in $M_{s}(E)$. Choose 
a holomorphic frame $\left(e_{1}, \ldots, e_{r}\right)$ of $E$ such that the flag $z^{0}$ is given by the basis $\left(e_{1}\left(x^{0}\right), \ldots, e_{r}\left(x^{0}\right)\right)$ (supposed to be orthonormal). Assume also $\left(e_{\lambda}\right)$ chosen such that $D e_{\lambda}\left(x^{0}\right)=0$ and consider the curvature tensor

$$
c(E)_{x^{0}}=\sum_{\substack{1 \leqslant j, k \leqslant n \\ 1 \leqslant \lambda, \mu \leqslant r}} c_{j k \lambda \mu} d x_{j} \wedge d x_{k} \otimes e_{\lambda}^{\star} \otimes e_{\mu}
$$

It can be shown that the associated curvature of $Q_{s}^{a}$ is

$$
c\left(Q_{s}^{a}\right)_{z^{0}}=\sum_{\substack{1 \leqslant j, k \leqslant n \\ 1 \leqslant \lambda \leqslant n}} a_{\lambda} c_{j k \lambda \lambda} d x_{j} \wedge d x_{k}+\sum_{a_{\lambda}>a_{\mu}}\left(a_{\lambda}-a_{\mu}\right) d z_{\lambda \mu} \wedge d \bar{z}_{\lambda \mu}
$$

where $\left(z_{\lambda \mu}\right)$ are the affine coordinates along the fiber $M_{s}\left(E_{x^{0}}\right)$. Finally, let $N(s)$ be the dimension of the fibers $M_{s}\left(E_{x}\right)$. Using the isomorphism (7.4), the strong Morse inequality becomes

$$
\sum_{m=0}^{q}(-1)^{q-m} \operatorname{dim} H^{m}\left(X, \Gamma^{k a} E \otimes F\right) \leqslant r^{\prime} \frac{k^{n+N(s)}}{(n+N(s)) !} \int_{Y\left(\leqslant q, Q_{s}^{a}\right)}\left(\frac{i}{2 \pi} c\left(Q_{s}^{a}\right)\right)^{n+N(s)} .
$$

The most interesting case is the case of symmetric powers $S^{k} E$. Then we simply have $M_{s}(E)=\mathbb{P}\left(E^{\star}\right), N(s)=r-1, Q_{s}^{a}=O_{E}(1)$.

\section{Related questions and open problems}

(a) As in the case of the Riemann-Roch Hirzebruch formula, it would be extremely interesting to get some insight on the error term $o\left(k^{n}\right)$ of the estimates. However, this problem encounters two major difficulties :

- First, the lower order terms in the asymptotic expansion of the heat kernel involve derivatives of the curvature of $E$, as well as terms coming from the curvature of $F$ or of the manifold $X$. These terms are very difficult to compute.

- Second, the open sets $X(\leqslant q, E)$ involved in the estimates may be extremely irregular, even when the metric of $E$ is smooth. This leaves very little hope of being able to handle the error term.

One way to avoid the difficulties of the first point is to assume that the dominant term is identically zero, i.e. that $i c(E)$ has rank $<n$ everywhere and is generically of a given rank $s$. Concerning the second point, a reasonable hypothesis is the following : assume that there is a smooth foliation $\mathcal{F}$ of codimension $s$ in $X$ such that $i c(E)$ vanishes along the leaves. With these hypotheses, Th. Bouche [Bou2] has shown that when $\operatorname{rk} E=\operatorname{rk} F=1, \ell \rightarrow+\infty, k / \ell \rightarrow+\infty$, then

$$
\begin{aligned}
& \sum_{0 \leqslant m \leqslant q}(-1)^{q-m} \operatorname{dim} H^{m}\left(X, E^{k} \otimes F^{\ell} \otimes G\right) \\
& \leqslant \frac{k^{s}}{s !} \frac{\ell^{n-s}}{(n-s) !}(\operatorname{rk} G) \int_{X(\leqslant q, E, F)}\left(\frac{i}{2 \pi} c(E)\right)^{s} \wedge\left(\frac{i}{2 \pi} c(F)\right)^{n-s}+o\left(k^{s} \ell^{n-s}\right),
\end{aligned}
$$


where the index set $X(m, E, F)$ is the set where $(i c(E))^{s} \wedge(i c(F))^{n-s}$ has sign $(-1)^{m}$. It would be very interesting to get rid of the tensor powers $F^{\ell}$, but then the difficulty is that the estimates cannot be localized along the leaves of the foliation.

(b) A natural problem is of course to extend the Morse inequalities to cohomology groups associated to other operators than $\bar{\partial}$. This has been carried out by Getzler [Ge3] for the operator $\bar{\partial}_{b}$ on a compact strongly pseudoconvex $C R$ manifold.

(c) Jean Varouchas has drawn my attention on the following interesting question of Fujiki related to the Grauert-Riemenschneider conjecture. The G-R conjecture was an attempt to characterize Moishezon manifolds, i.e. manifolds which have a projective algebraic modification.

Another interesting class of manifolds is the Fujiki class $\mathcal{C}$, that is, the class of compact complex manifolds which have a Kähler modification (for instance, such manifolds possess Hodge decomposition). Assume that $X$ is compact and has a closed semi-positive $(1,1)$-form $\omega$ such that $\omega$ is positive definite on a dense open subset (say, on the complement of an analytic subset). Then, is $X$ in the Fujiki class $\mathcal{C}$ ?

\section{References}

[A-G] Andreotti A. and Grauert H. - Théorèmes de finitude pour la cohomologie des espaces complexes, Bull. Soc. Math. France, 90 (1962), 193-259.

[A-B-P] AtiYAh M.F., Bott R. and Patodi V.K. - On the heat equation and the index theorem, Invent. Math., 19 (1973), 279-330.

[A-S] Atiyah M.F. and Singer I.M. - The index of elliptic operators III, Ann. of Math., 87 (1968), 546-604.

[Bi] Bismut J.M. - Demailly's asymptotic inequalities : a heat equation proof, J. Funct. Analysis, 72 (1987), 263-278.

[Bot] BotT R. - Homogeneous vector bundles, Ann. of Math., 66 (1957), 203-248.

[Bou1] Bouche T. - Inégalités de Morse pour la $d^{\prime \prime}$-cohomologie sur une variété holomorphe non compacte, to appear in Ann. Sci. Ec. Norm. Sup., 1989.

[Bou2] Bouche T. - Sur les inégalités de Morse holomorphes lorsque la courbure du fibré en droites est dégénérée, Submitted to Ann. Scuola Norm. Sup. Pisa Cl. Sci. IV, to appear.

[De1] Demailly J.P. - Sur l'identité de Bochner-Kodaira-Nakano en géométrie hermitienne, Sém. P. Lelong, P. Dolbeault, H. Skoda (Analyse), 1983/84, Lecture Notes in Mathematics, 1198 (1986), 88-97.

[De2] Demailly J.P. - Champs magnétiques et inégalités de Morse pour la $d^{\prime \prime}$-cohomologie, C. R. Acad. Sci., 301 (1985), 119-122 and Ann. Inst. Fourier, 35 (1985), 185-229.

[De3] Demailly J.P. - Une preuve simple de la conjecture de Grauert-Riemenschneider, Sém. P. Lelong, P. Dolbeault, H. Skoda (Analyse), 1985/86, Lecture Notes in Mathematics, 1295 (1987), 48-58.

[Ge1] GetZleR E. - Pseudodifferential operators on supermanifolds and the Atiyah-Singer index theorem, Comm. Math. Phys., 92 (1983), 167-178. 
[Ge2] GetzleR E. - Inégalités asymptotiques de Demailly pour les fibrés vectoriels, C. R. Acad. Sci., 304 (1987), 475-478.

[Ge3] GetzLER E. - An analogue of Demailly's inequality for strictly pseudoconvex $C R$ manifolds, J. Diff. Geom., 29 (1989), 231-244.

[G-R] GRAUERT H. und RIEMEnsChneIDER O. - Verschwindungssätze für analytische Kohomologiegruppen auf Komplexe Raüme, Invent. Math., 11 (1970), 263-292.

[Ji] JI S. - Inequality for distortion function of invertible sheaves on abelian varieties, Duke Math. J., 58 (1989), 657-667.

[Kem] KEMPF G. - Metric on invertible sheaves on abelian varieties, preprint 1988, Johns Hopkins Univ. in Baltimore.

[Ram] RAMACHANDRAN M. - Private communication, July1989.

[Sie] SiEgEL C.L. - Meromorphe Funktionen auf kompakten Mannigfaltigkeiten, Nachr. Akad. Wiss. Göttingen Math. Phys. K., 4 (1955), 71-77.

[Siu1] SIU Y.T. - A vanishing theorem for semi-positive line bundles over non-Kähler manifolds, J. Differential Geometry, 19 (1984), 431-452.

[Siu2] SIU Y.T. - Some recent results in complex manifold theory related to vanishing theorems for the semi-positive case, Proceedings of the Math. Arbeitstagung 1984, Lecture Notes in Mathematics, 1111 (1985), 169-192.

[Siu3] SIU Y.T. - Asymptotic Morse inequalities for analytic sheaf cohomology, Sém. Bourbaki, exposé $\mathrm{n}^{\mathrm{o}}, \mathbf{6 6 6}$ (juin1986), 16p.

[Siu4] SIU Y.T. - Calculus inequalities derived from holomorphic Morse inequalities, Preprint Harvard University, 1986, Math. Annalen (around 1990).

[Ti] TIAN G. - Kähler metrics on algebraic manifolds, PhD Thesis, Harvard University,1988.

[Wi] Witten E. — Supersymmetry and Morse theory, J. Diff. Geom., 17 (1982), 661-692.

\section{Institut Fourier}

B.P.74

38402 ST MARTIN D'HÈRES Cedex

(France)

(31 mai 2007) 